\title{
SYMMETRY-ADAPTED BASIS SETS
}

Automatic Generation for Problems in Chemistry and Physics 
This page intentionally left blank

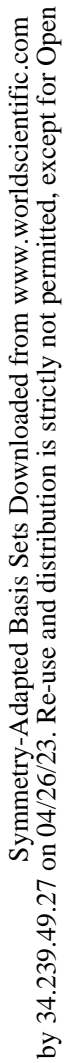




\title{
SYMMETRY-ADAPTED BASIS SETS
}

Automatic Generation for Problems in Chemistry and Physics

\author{
John Scales Avery \\ Sten Rettrup \\ University of Copenhagen, Denmark \\ James Emil Avery \\ NZIAS, Massey University, New Zealand
}


Published by

World Scientific Publishing Co. Pte. Ltd.

5 Toh Tuck Link, Singapore 596224

USA office: 27 Warren Street, Suite 401-402, Hackensack, NJ 07601

UK office: 57 Shelton Street, Covent Garden, London WC2H 9HE

\section{British Library Cataloguing-in-Publication Data}

A catalogue record for this book is available from the British Library.

\section{SYMMETRY-ADAPTED BASIS SETS \\ Automatic Generation for Problems in Chemistry and Physics}

Copyright (C) 2012 by World Scientific Publishing Co. Pte. Ltd.

All rights reserved. This book, or parts thereof, may not be reproduced in any form or by any means, electronic or mechanical, including photocopying, recording or any information storage and retrieval system now known or to be invented, without written permission from the Publisher.

For photocopying of material in this volume, please pay a copying fee through the Copyright Clearance Center, Inc., 222 Rosewood Drive, Danvers, MA 01923, USA. In this case permission to photocopy is not required from the publisher.

ISBN-13 978-981-4350-46-4

ISBN-10 981-4350-46-X

Printed in Singapore. 


\section{Contents}

Preface $\quad$ ix

1. GENERAL CONSIDERATIONS 1

1.1 The need for symmetry-adapted basis functions . . . . . . . . . 1

1.2 Fundamental concepts . . . . . . . . . . . . . 2

1.3 Definition of invariant blocks . . . . . . . . . . . 3

1.4 Diagonalization of the invariant blocks . . . . . . . . 5

1.5 Transformation of the large matrix to block-diagonal

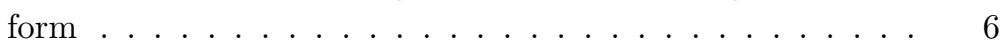

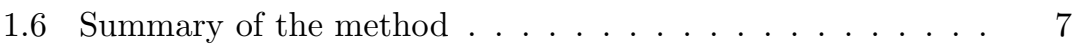

2. EXAMPLES FROM ATOMIC PHYSICS 9

2.1 The Hartree-Fock-Roothaan method for calculating

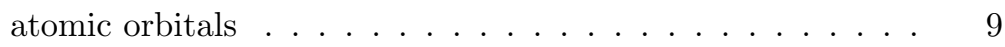

2.2 Automatic generation of symmetry-adapted configurations . 12

2.3 Russell-Saunders states . . . . . . . . . . . . . . . . . 14

2.4 Some illustrative examples . . . . . . . . . . . . . . . 15

2.5 The Slater-Condon rules . . . . . . . . . . . . . . . . 18

2.6 Diagonalization of invariant blocks using the Slater-Condon rules . . . . . . . . . . . . . 20

3. EXAMPLES FROM QUANTUM CHEMISTRY 27

3.1 The Hartree-Fock-Roothaan method applied to molecules . . . . . . . . . . . . . . 27

3.2 Construction of invariant subsets . . . . . . . . . . . . 28

3.3 The trigonal group $\mathrm{C}_{3 v}$; the $\mathrm{NH}_{3}$ molecule . . . . . . 30 
4. GENERALIZED STURMIANS APPLIED TO ATOMS 33

4.1 Goscinskian configurations . . . . . . . . . . . . . 33

4.2 Relativistic corrections . . . . . . . . . . . . . . . 38

4.3 The large- $Z$ approximation: Restriction of the basis set to

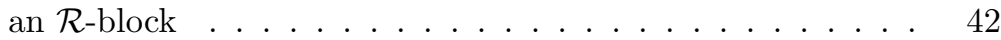

4.4 Electronic potential at the nucleus in the large- $Z$

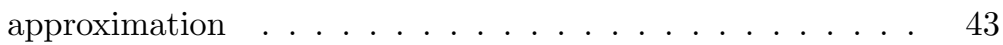

4.5 Core ionization energies . . . . . . . . . . . . 46

4.6 Advantages and disadvantages of Goscinskian configurations 49

$4.7 \mathcal{R}$-blocks, invariant subsets and invariant blocks . . . . . . 50

4.8 Invariant subsets based on subshells; Classification according to $M_{L}$ and $M_{s} \ldots \ldots \ldots \ldots \ldots \ldots \ldots$

4.9 An atom surrounded by point charges . . . . . . . 62

5. MOLECULAR ORBITALS BASED ON STURMIANS 71

5.1 The one-electron secular equation . . . . . . . . . . 71

5.2 Shibuya-Wulfman integrals and Sturmian overlap integrals evaluated in terms of hyperpherical harmonics . . . . . . . 78

5.3 Molecular calculations using the isoenergetic configurations 85

5.4 Building $T_{\nu^{\prime} \nu}^{(N)}$ and $\mathfrak{S}_{\nu^{\prime} \nu}^{(N)}$ from 1-electron components . . . 87

5.5 Interelectron repulsion integrals for molecular Sturmians from hyperspherical harmonics . . . . . . . . . . 88

5.6 Many-center integrals treated by Gaussian expansions (Appendix E) . . . . . . . . . . . . . . . . . 93

5.7 A pilot calculation . . . . . . . . . . . . . 97

5.8 Automatic generation of symmetry-adapted basis functions 98

6. AN EXAMPLE FROM ACOUSTICS 101

6.1 The Helmholtz equation for a non-uniform medium . . . . . 101

6.2 Homogeneous boundary conditions at the surface of a cube 101

6.3 Spherical symmetry of $v(\mathbf{x})$; nonseparability of the Helmholtz equation . . . . . . . . . . . . . . . . 103

6.4 Diagonalization of invariant blocks . . . . . . . . . 105

7. AN EXAMPLE FROM HEAT CONDUCTION 111

7.1 Inhomogeneous media . . . . . . . . . . . . . . . . 111

7.2 A 1-dimensional example . . . . . . . . . . . . . . 112

7.3 Heat conduction in a 3-dimensional inhomogeneous medium 114 
8. SYMMETRY-ADAPTED SOLUTIONS BY ITERATION

8.1 Conservation of symmetry under Fourier transformation . . 121

8.2 The operator $-\Delta+p_{\kappa}^{2}$ and its Green's function . . . . . . . 122

8.3 Conservation of symmetry under iteration of the Schrödinger equation . . . . . . . . . . . . . . 123

8.4 Evaluation of the integrals . . . . . . . . . . . . . . 125

8.5 Generation of symmetry-adapted basis functions by iteration 127

8.6 A simple example . . . . . . . . . . . . . . . . . 128

8.7 An alternative expansion of the Green's function that applies to the Hamiltonian formulation of physics . . . . . 129

Appendix A REPRESENTATION THEORY OF FINITE GROUPS 131

A.1 Basic definitions . . . . . . . . . . . . . . . . . 131

A.2 Representations of geometrical symmetry groups . . . . . 132

A.3 Similarity transformations . . . . . . . . . . . . . 133

A.4 Characters and reducibility . . . . . . . . . . . 134

A.5 The great orthogonality theorem . . . . . . . . 136

A.6 Classes . . . . . . . . . . . . . . . . . . . . . . . . . 141

A.7 Projection operators . . . . . . . . . . . . . . . 142

A.8 The regular representation . . . . . . . . . . . . . . . . . 144

A.9 Classification of basis functions . . . . . . . . . . . . 146

$\begin{array}{lll}\text { Appendix B STURMIAN BASIS SETS } & 149\end{array}$

B.1 One-electron Coulomb Sturmians . . . . . . . . . . . . . 149

B.2 Löwdin-orthogonalized Coulomb Sturmians . . . . . . . . . 151

B.3 The Fock projection . . . . . . . . . . . . . 153

B.4 Generalized Sturmians and many-particle problems . . . . . 154

B.5 Use of generalized Sturmian basis sets to solve the manyparticle Schrödinger equation . . . . . . . . . . . . 155

B.6 Momentum-space orthonormality relations for Sturmian basis sets . . . . . . . . . . . . . 156

B.7 Sturmian expansions of $d$-dimensional plane waves $\ldots \ldots .158$

B.8 An alternative expansion of a $d$-dimensional plane wave . . 159

Appendix C ANGULAR AND HYPERANGULAR INTEGRATION 161

C.1 Monomials, homogeneous polynomials, and harmonic polynomials . . . . . . . . . . . . . . 161

C.2 The canonical decomposition of a homogeneous polynomial 162 
C.3 Harmonic projection . . . . . . . . . . . . . . . . 163

C.4 Generalized angular momentum . . . . . . . . . . . . . . . 164

C.5 Angular and hyperangular integration . . . . . . . . . 165

C.6 An alternative method for angular and hyperangular integrations . . . . . . . . . . . . . . 168

C.7 Angular integrations by a vector-pairing method . . . . . 170

Appendix D INTERELECTRON REPULSION INTEGRALS 173

D.1 The generalized Slater-Condon rules _ . . . . . . . . . 173

D.2 Separation of atomic integrals into radial and angular parts 174

D.3 Evaluation of the radial integrals in terms of hypergeometric functions . . . . . . . . . . . . . . 175

D.4 Evaluation of the angular integrals in terms of CondonShortley coefficients . . . . . . . . . . . . . . 177

Appendix E GAUSSIAN EXPANSION OF MOLECULAR STURMIANS

E.1 Expansions of Coulomb Sturmian densities in terms of Gaussians . . . . . . . . . . . . . . . 179

Appendix F EXPANSION OF DISPLACED FUNCTIONS IN TERMS OF LEGENDRE POLYNOMIALS 185

F.1 Displaced spherically symmetric functions . . . . . . . . . 185

F.2 An alternative method . . . . . . . . . . . . . 186

F.3 A screened Coulomb potential . . . . . . . . . . . . 187

F.4 Expansion of a displaced Slater-type orbital . . . . . . . . 188

F.5 A Fourier transform solution . . . . . . . . . . . . 190

F.6 Displacement of functions that do not have spherical symmetry . . . . . . . . . . . . . . 193

Appendix G MULTIPOLE EXPANSIONS 195

$\begin{array}{lll}\text { Appendix H HARMONIC FUNCTIONS } & 199\end{array}$

H.1 Harmonic functions for $d=3 \ldots \ldots \ldots$. . . . . . 199

H.2 Spaces of higher dimension _. . . . . . . . . . 200

$\begin{array}{ll}\text { Bibliography } & 207\end{array}$ 


\section{Preface}

In a number of previous papers and a book [Avery and Avery, 2006], two of us introduced the Generalized Sturmian Method applied to atoms. One of the interesting features of this method is that it leads to a secular equation of the form

$$
\sum_{\nu}\left[\delta_{\nu^{\prime}, \nu} Z \mathcal{R}_{\nu}+T_{\nu^{\prime}, \nu}^{\prime}-p_{\kappa} \delta_{\nu^{\prime}, \nu}\right] C_{\nu, \kappa}=0
$$

where $Z$ is the nuclear charge and where $p_{\kappa}$ is not the energy but is a scaling factor related to the energy of an atomic state by

$$
E_{\kappa}=-\frac{p_{\kappa}^{2}}{2}
$$

In equation (0.1),

$$
\mathcal{R}_{\nu} \equiv \sqrt{\frac{1}{n^{2}}+\frac{1}{n^{\prime 2}}+\frac{1}{n^{\prime \prime 2}}+\ldots}
$$

$\nu$ being a collection of indices that label a configuration (a Slater determinant) and $n, n^{\prime}, n^{\prime \prime}, \ldots$ being the principal quantum numbers of the atomic spin-orbitals that appear in the configuration. The matrix $T_{\nu^{\prime}, \nu}^{\prime}$ represents the effects of interelectron repulsion. If interelectron repulsion is entirely neglected, the energy reduces to

$$
E_{0}=-\frac{1}{2}\left(Z \mathcal{R}_{\nu}\right)^{2}=-\frac{1}{2} Z^{2}\left(\frac{1}{n^{2}}+\frac{1}{n^{\prime 2}}+\frac{1}{n^{\prime \prime 2}}+\ldots\right)
$$

that is to say, it reduces to the energy of a set of completely independent electrons moving in the attractive Coulomb potential of the nucleus. We introduced a large- $\mathrm{Z}$ approximation, in which the basis set is restricted to configurations all of which correspond to the same value of $R_{\nu}$, and we found that in this approximation, the energy of an atomic state is given by

$$
E_{\kappa} \approx-\frac{1}{2}\left(Z \mathcal{R}_{\nu}-\left|\lambda_{\kappa}\right|\right)^{2}
$$


where $\lambda_{\kappa}$ is a root of the energy-independent interelectron repulsion matrix $T_{\nu^{\prime}, \nu}^{\prime}$. We found that for an isoelectronic series corresponding to a fixed number of electrons $N$, the large-Z approximation approached the experimental data more and more closely with increasing $Z$, provided that a simple correction was made for relativistic effects. We also found that the eigenfunctions of the simplified secular equation

$$
\sum_{\nu}\left[T_{\nu^{\prime}, \nu}^{\prime}-\lambda_{\kappa} \delta_{\nu^{\prime}, \nu}\right] C_{\nu, \kappa}=0
$$

were Russell-Saunders states, i.e. simultaneous eigenfunctions of the total angular momentum operator $L^{2}$, its $z$-component $L_{z}$, the total spin operator $S^{2}$, and its $z$-component $S_{z}$. These Russell-Saunders states could be used as symmetry-adapted basis functions for more refined calculations, where the large- $Z$ approximation was abandoned. This automatic generation of symmetry-adapted basis functions proved to be a great convenience.

In the present book, we are joined by Lektor Sten Rettrup, who has taught Group Theory and Quantum Chemistry for many years at the University of Copenhagen. The book results from a wish to go more deeply into the mathematical reasons behind the automatic generation of symmetryadapted basis sets, which we observed when applying the Generalized Sturmian Method to atoms in the large- $Z$ approximation. We believe that the same principles can be used in many problems encountered in physics and chemistry, where the size of the basis sets required for accurate solutions makes it desirable to use symmetry-adapted basis functions.

The first chapter discusses the general mathematical principles that underlie the automatic generation of symmetry-adapted basis functions, and subsequent chapters aim at developing and illustrating these basis principles. In Chapter 2, the principles are applied to atomic Hartree-Fock calculations followed by Configuration Interaction computations, while in Chapter 3, this is extended to molecules. As is well known, the number of configurations needed for accurate results in CI calculations is often extremely large - often in the millions. Therefore reducing this number by means of symmetry-adapted basis functions is highly desirable. We hope that Chapters 2 and 3 indicate paths one might follow to do this.

Chapter 4 reviews and extends our studies of the Generalized Sturmian method applied to atoms. Among the new topics not discussed previously, is the calculation of the effect on atoms when they are surrounded by symmetrical arrays of point charges. This effect is calculated using the Generalized Sturmian Method. 
Chapter 5 sketches the extension of the Generalized Sturmian Method to molecules. Coulomb Sturmians have long been used as basis functions for solution for a single electron moving in the attractive field of two or more nuclei. Among the important pioneers in this field are C.E. Wulfman, V. Aquilanti and T. Koga. In Chapter 5, we try to show how Sturmian basis functions can be used in $N$-electron molecules. To do this, we need to evaluate many-center interelectron repulsion integrals involving Coulomb Sturmians, which are exponential-type orbitals. We propose a new method for evaluating these integrals making use of the Fock projection, which maps Coulomb Sturmians onto sets of 4-dimensional hyperspherical harmonics. The properties of hyperspherical harmonics are then used to evaluate the needed integrals.

In Chapters 6 and 7, we attempt to show how the basic principles for automatic generation of symmetry-adapted basis functions may be applied to other areas of physics. We hope that readers will find ways of applying these general principles to their own special areas of research. Chapter 8 discusses how symmetry-adapted basis functions can be generated by iteration.

Atomic units are used throughout this book: All lengths are in Bohrs and all energies are in Hartrees.

Programs for carrying out calculations of the type discussed in this book can be found at our website [Avery and Avery, 2006a]

http://sturmian.kvante.org/symmetry

These programs may be freely downloaded and modified for general use.

John Scales Avery, Sten Rettrup and James Emil Avery

Copenhagen, 2011 